\title{
The effects of endotracheal intubation via Mc-Grath Videolaryngoscope on intraocular pressure
}

\author{
Ozkan A.S. ${ }^{1}$, Akbas S. ${ }^{1}$, Polat N. ${ }^{2}$, Yalın M.R. ${ }^{1}$ \\ ${ }^{1}$ Inonu University, Dept of Anaesthesiology, Malatya, Turkey, \\ 2Inonu University, Dept of Ophthalmology, Malatya, Turkey
}

Background and Goal of Study: In this study; we aimed to compare the effects of endotracheal intubation via direct laryngoscope(DLS) and Mc-Grath Videolaryngoscope(VL) on intraocular pressure. Materials and Methods: Total of 50 ASA (American Society of Anesthesiologist) Grade 1-2, Mallampati score 1 or 2, age between 18 to 65 patient planned to undergo nonophtalmic surgery included to study. Patients with glaucoma, diabetes mellitus, cardiovascular and pulmonary diseases, ASA Grade III and IV, BMI more than 35, difficult intubation, undergo obstetrical surgery and propofol, fentanyl, rocuronium contraindicated were excluded from the study. Patients divided randomly into 2 groups as direct laryngoscopic and videolaryngoscopic intubation group. Patients were preoxigenated with $\% 100 \mathrm{O} 2$ for 3 minutes then anesthesia was induced using propofol 2 $\mathrm{mg} / \mathrm{kg}$, fentanyl $1 \mathrm{mcg} / \mathrm{kg}$, and rocuronium $0,5 \mathrm{mg} / \mathrm{kg}$ in both groups. After 3 minutes mask ventilation, patients were intubated (women with No:7-7,5, men with No:8-8,5 intubation tube) by oral route. SBP, DBP, MAP, SpO2, PI recorded and IOP (intraocular pressure) measured by ophthalmologist by tonopen were recorded preinduction (basal), after induction, 1,2,3,5 minutes after intubation, respectively. Period between handling of laryngoscope or videolaryngoscope after termination of mask ventilation and obtain end tidal $\mathrm{CO} 2$ was accepted as application time and recorded. Study terminated after 5th minute values taken. Results and Discussion: There was no statistically significant difference in distirubiton of sex, weight, age, height, BMI, MPS and ASA between groups. Duration of intubation in VL group was $32 \pm 2$ sn and statistically significantly longer then DLS group $(23,8 \pm 2,9)$ $(\mathrm{p}<0,05)$. Statistically significant increase was found in intraocular pressure after 1 minute intubation in DLS group $(16,1 \pm 2,4)$ compared with VL group $(12,1 \pm 2,5) \quad(\mathrm{p}<\quad 0,001)$. Conclusion(s): We concluded that endotracheal intubation by McGrath videolaryngoscope could be more useful with regard to endotracheal intubation by DLS in patients with high IOP patologies when performed by experienced anesthesiologists. 